This is the post peer-review accepted manuscript of:

A. Mariani, A. Giorgetti and M. Chiani, "Robust Detection with Low-Complexity SDRs:

A Pragmatic Approach," 2018 IEEE 29th Annual International Symposium on Personal, Indoor and Mobile Radio Communications (PIMRC), Bologna, 2018, pp. 16.

The published version is available online at:

https://doi.org/10.1109/PIMRC.2018.8580886

(C) 2018 IEEE. Personal use of this material is permitted. Permission from IEEE must be obtained for all other uses, in any current or future media, including reprinting/republishing this material for advertising or promotional purposes, creating new collective works, for resale or redistribution to servers or lists, or reuse of any copyrighted component of this work in other works. 


\title{
Robust Detection with Low-Complexity SDRs: A Pragmatic Approach
}

\author{
Andrea Mariani, Andrea Giorgetti, and Marco Chiani
}

\begin{abstract}
The increasing availability of inexpensive software defined radios (SDRs) allows nowadays to implement cognitive radio (CR) functionalities in large scale networks such as the Internet-of-Things and future 5G systems. In this work, we focus on the spectrum sensing functionality that must take into account the front-end impairments of low-cost devices. Based on the noise model of a real SDR dongle, we address the problem of robust signal detection in the presence of noise power uncertainty and non-flat noise power spectral density (PSD). In particular, we analyze the receiver operating characteristic (ROC) of different known detectors in the presence of such front-end impairments, to understand the performance attainable in a real-world scenario. Based on the analysis, we propose two frequency-domain detectors that are proven to outperform previously proposed spectrum sensing techniques such as, e.g., eigenvalues-based tests.
\end{abstract}

\section{INTRODUCTION}

The increasing demand for a limited resource such as the radio-frequency (RF) spectrum is the propelling force toward new ways different radio ecosystems can coexist. In this context, we are witnessing a prime example of the need for coexistence between radar and wireless communications, a topic which received increasing attention by the DARPA and the US National Spectrum Consortium [1].

Software defined radio (SDR) was conceived with the aim of building flexible front-ends for transceivers in which radio functionalities are controlled and programmable by software [2]. Even if the concept of SDR is known from the $80 \mathrm{~s}$, its actual diffusion started in the second half of the 90s with first application in the military context and more recently for implementing multi-band multi-standard platforms, in particular, in the context of cognitive radio (CR) [3]-[5]. In the last few years we have seen the development and diffusion of several high-performance SDRs such as universal software radio platforms (USRPs) [6]. These equipments can be used for implementing a wide range of applications, but their cost, ranging from hundreds to thousands of dollars, makes them prohibitively expensive for simple devices and large-scale networks. This

This work was supported in part by the European Project EuroCPS through the H2020 Framework under Grant 644090. A. Giorgetti and M. Chiani are with National Inter-University Consortium for Telecommunications (CNIT) and DEI, University of Bologna, Via Venezia 52, 47521 Cesena (FC), Italy, Email: \{a.mariani, andrea.giorgetti, marco.chiani\}@unibo.it. aspect is further exacerbated by the emerging idea of a globally interconnected continuum of low-cost devices and things, namely the Internet-of-Things (IoT). An example of cheap devices which appear more suitable for these contexts are low-cost low-complexity SDRs, such as digital video broadcasting - terrestrial (DVB-T) dongles, based on the Realtek RTL2832U chipset. ${ }^{1}$ Such devices, referred as RTL-SDR dongles, cost just few dollars and are already used to implement low-cost receivers [7].

The adoption of low-cost devices is, on the other hand, critical for spectrum sensing (SS) due to the presence of receiver nonidealities which have a strong impact on the detection performance, especially at very low signal-tonoise ratios (SNRs) [8], [9]. It is therefore fundamental to adopt a proper receiver characterization of the SDR front-end and to design robust detection strategies. In particular, RTL-SDR devices are characterized by a nonwhite noise power spectral density (PSD), which is mainly caused by filtering in the receiver chain [10][12]. Another practical impairment in all receivers is the presence of noise power fluctuations (caused e.g., by receiver temperature variations) often referred as noise uncertainty [13]-[15].

The noise uncertainty problem can be counteracted adopting noise power estimation strategies [13], [16], [17]. An alternative approach adopts detection metrics which are independent on the noise power level, and in single antenna receivers oversampling is the key element. In fact, when a signal is present in the observed frequency band, oversampling implies that the received samples are not independent. Time-domain tests that detect the presence of correlation among the received samples have been proposed in particular in the context of eigenvalue-based detection [11], [12], [18]-[20]. Other impairments, such as in-phase and quadrature-phase imbalance, low-noise amplifier nonlinearities, phase noise, and aliasing may cause detrimental effects if not properly accounted and counteracted [21][23].

In this paper, we discuss the adoption of RTL-SDR receivers as a platform to implement spectrum sensing

${ }^{1}$ The RTL2832U chipset is a high-performance DVB-T receiver that implements a COFDM demodulator and supports a USB 2.0 interface. 
TABLE I

OCCURRENCE RATE OF GAUSSIANITY TESTS FOR I-Q SAMPLES CAPTURED WITH A RTL-SDR RECEIVER. TESTS ARE BASED ON THE OBSERVATION OF 1000 SAMPLES, EACH TEST IS PERFORMED 50000 TIMES WITH A SIGNIFICANCE LEVEL OF 0.05 .

\begin{tabular}{c||c|c} 
test & real part & imaginary part \\
\hline Anderson-Darling (Gaussianity) & 0.965 & 0.966 \\
\hline Andersson-Perlman (Circularity) & \multicolumn{2}{|c}{0.999} \\
\hline
\end{tabular}

functionalities for signal detection and thereby seeking spectrum holes. More precisely, we propose two frequency-domain detectors and show that they outperform commonly adopted eigenvalue-based strategies which are deemed the most effective. We study, in particular, a frequency-domain version of the energy detector (ED) with estimated noise power (ENP) [13]. We also propose a detector which metric is the correlation coefficient between the estimated PSD of the received signal and a reference noise PSD obtained by an off-line calibration phase. Our pragmatic approach, driven by real waveforms, revealed the most dominant impairments in low-cost devices and proposed robust detectors with good performance.

Throughout the paper, boldface letters denote matrices and vectors; $\mathbf{I}_{m}$ represents the identity matrix; $(\cdot)^{\mathrm{T}}$ and $(\cdot)^{\mathrm{H}}$ stand for transposition and hermitian transposition, respectively; the $p$-norm of the vector $\mathbf{v}$ is $\|\mathbf{v}\|_{p} \triangleq$ $\left(\sum_{i}\left|v_{i}\right|^{p}\right)^{1 / p}$, where $v_{i}$ is the $i$ th element of $\mathbf{v} ; \mathbb{E}\{\cdot\}$ denotes the expectation operator; $\lfloor x\rfloor$ denotes largest integer less than or equal to $x$.

\section{System MODEl AND Noise ANAlysis}

The detection task is to distinguish the presence or absence of any signal in the observed band. The two hypotheses are denoted, respectively, by $\mathcal{H}_{1}$ and $\mathcal{H}_{0}$. After downconversion and sampling, the $N$-length vector of received samples is given by ${ }^{2}$

$$
\mathbf{y}= \begin{cases}\mathbf{s}+\mathbf{n}, & \mathcal{H}_{1} \\ \mathbf{n}, & \mathcal{H}_{0}\end{cases}
$$

where $\mathbf{n}$ denotes noise and $\mathbf{s}$ contains the transmitted signal samples including channel effects. Oversampling is implemented using a sampling rate $f_{\mathrm{s}}=\mathrm{OSF} \cdot B$, where $B$ is the bandwidth of the signal to be detected and OSF is the integer oversampling factor (OSF) [11], [24]. Without loss of generality we assume that both $\mathbf{s}$ and $\mathbf{n}$ are modeled as vectors of zero mean random variables (r.v.s).

The most common assumption in communication literature is to model noise as a white Gaussian process. When dealing with a real system, especially a lowcost device, it is important to characterize noise and identify a suitable model. Concerning the Gaussianity of

\footnotetext{
${ }^{2}$ In the paper we always consider column vectors.
}

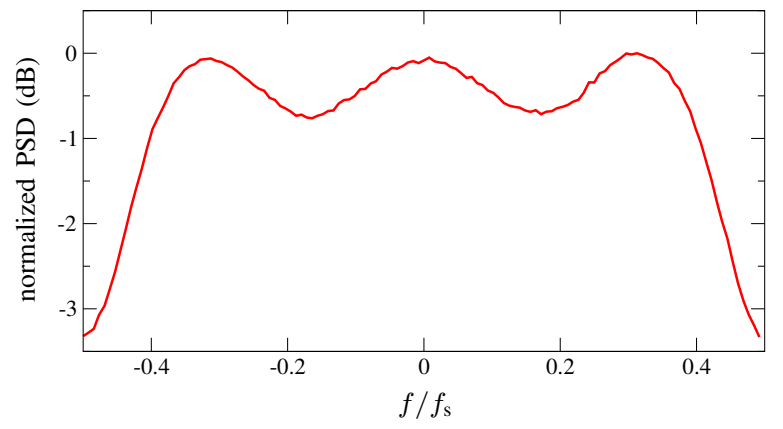

Fig. 1. Example of a spurious-free noise PSD shape measured in a RTL-SDR dongle. This normalized PSD has been estimated from samples, under $\mathcal{H}_{0}$ hypothesis, using Bartlett's periodogram with $N_{\text {fft }}=128$ and $N_{\text {avg }}=1000$.

noise, we tested noise-only samples captured using RTLSDR dongles using the Anderson-Darling test, which can be adopted when the mean value and the variance are not known [25]-[27]. The tests show that the captured samples well fit a Gaussian distribution, with about 0.95 probability. Beyond normality we also tested the circularity property of the samples using the AnderssonPerlman test [28], [29], obtaining a probability close to 1. Results of both Gaussianity and circularity tests are reported in Table I.

Regarding the whiteness assumption, we estimated the PSD in the absence of an input signal. Except for a multiplying constant (i.e., a vertical shift in $\mathrm{dB}$ scale), the characteristic shape of the noise PSD is shown in Fig. 1. This shape can be ascribed to the digital filter in the decimation stage of the RTL-SDR receiver chain [10]-[12]. Based on this analysis we model noise as a Gaussian process with correlated samples. More precisely, we express the noise sample vector as $\mathbf{n}=\sigma \widetilde{\mathbf{n}}$, where $\widetilde{\mathbf{n}}$ is composed by zero mean Gaussian samples with unity variance. The noise power $\sigma^{2}=\mathbb{E}\left\{\mathbf{n}^{\mathrm{H}} \mathbf{n}\right\}$ is an unknown time-varying parameter which accounts for power fluctuations, also known as noise power uncertainty [13]-[15]. However, its variations are generally slow, and thus $\sigma$ can be considered constant for the $N$ collected samples [14].

We would like to remark that the focus of the paper is signal detection in presence of non-flat noise spectral density and noise power uncertainty. Although of interest, we do not consider the problem of detection in presence of spurs, caused by harmonics from mixer, local oscillator leakage, DC offset, etc. [30], [31], which will be object of further investigations. Therefore, in this work we analyze samples captured in bands in which no spurs have been observed. This assumption is sometimes verified in practice also when spectrum sensing is preceded by spurious removal like, e.g., an upstream spur detection and spur exclusion stage [30]. 


\section{A. Calibration}

The detection tests proposed in the next sections exploit the knowledge of the second-order statistical properties of receiver noise, although noise power remains unknown. We consider, therefore, that detection is preceded by a off-line calibration stage (under hypothesis $\mathcal{H}_{0}$ ), in which the system estimates either the noise PSD, $\mathcal{W}(f)$, for frequency-domain detectors, or the noise covariance matrix, $\boldsymbol{\Sigma}_{0}$, for time-domain detectors. This can be done, e.g., replacing the antenna with a $50 \Omega$ load, and estimating the PSD or the covariance matrix from the collected samples.

In both cases, due to noise uncertainty, the noise PSD and the covariance matrix are known except for a multiplicative factor related to the time-varying noise power.

\section{FREQUENCY-DOMAIN DETECTORS}

Frequency-domain spectrum sensing is based on the estimation of a frequency representation of received samples and adoption of a test to infer the presence or absence of a signal. For simplicity, the frequencydomain representation is based on the PSD estimation through the averaged periodogram (also known as Bartlett's periodogram), which is based on a $N_{\text {fft-points }}$ discrete Fourier transform (DFT) [32, Section 12.2.1]. In particular, assuming that the total number of samples is $N=N_{\mathrm{fft}} \cdot N_{\mathrm{avg}}$, the $i$ th element of the averaged periodogram $\boldsymbol{P}$ is computed as [32]

$$
P_{i}=\frac{1}{N_{\mathrm{fft}} N_{\mathrm{avg}}} \sum_{k=1}^{N_{\mathrm{avg}}}\left|\sum_{m=1}^{N_{\mathrm{fft}}} y_{m+(k-1) N_{\mathrm{fft}}} e^{-j 2 \pi \frac{i m}{N_{\mathrm{fft}}}}\right|^{2}
$$

where $y_{n}$ is the $n$th element of the received vector $\mathbf{y}$.

The Bartlett's periodogram can be used also to estimate the noise PSD during the calibration phase. In the following, we denote with $\boldsymbol{W}$ the vector of length $N_{\mathrm{fft}}$ containing the estimate of the noise PSD, $\mathcal{W}(f)$, obtained by means of (2), i.e.,

$$
W_{i}=\frac{1}{N_{\mathrm{fft}} N_{\mathrm{avg}}} \sum_{k=1}^{N_{\text {avg }}}\left|\sum_{m=1}^{N_{\mathrm{fft}}} n_{m+(k-1) N_{\mathrm{fft}}} e^{-j 2 \pi \frac{i m}{N_{\mathrm{fft}}}}\right|^{2} .
$$

The original implementation of the ED considers the received signal power as a test statistic. Thus, the frequency domain version of the $\mathrm{ED}$ is given by $\mathrm{y}^{3,4}$

$$
\mathrm{T}_{\text {ed-all }}=\|\boldsymbol{P}\|_{1} \underset{\mathcal{H}_{0}}{\stackrel{\mathcal{H}_{1}}{\gtrless}} \xi .
$$

Considering oversampling, the signal band $\mathcal{B}=\left[f_{\mathrm{L}}, f_{\mathrm{H}}\right]$ is smaller than the observed frequency band, i.e., denoting with $f_{\mathrm{L}}$ and $f_{\mathrm{H}}$ the lower and higher baseband signal

\footnotetext{
${ }^{3}$ Note that by Parseval's theorem $\mathrm{T}_{\text {ed-all }}=\frac{1}{N_{\text {avg }}} \sum_{i} \mathbf{y}_{i}{ }^{\mathrm{H}} \mathbf{y}_{i}$, which is proportional to the usual ED metric [13].

${ }^{4}$ In the paper $\xi$ denotes any detection threshold.
}

frequencies, respectively, then $-f_{\mathrm{s}} / 2 \leq f_{\mathrm{L}}<f_{\mathrm{H}} \leq$ $f_{\mathrm{s}} / 2$. Thus, implementing the ED as in (4), we include in the decision metric the noise-only contributions out of the signal band. It is therefore reasonable to modify the ED metric including only the frequency components that may contain the signal. Denoting as $\boldsymbol{P}_{[\mathcal{B}]}$ the vector that contains the periodogram bins for $f_{\mathrm{L}} \leq f \leq f_{\mathrm{H}}$, we derive the test

$$
\mathrm{T}_{\text {ed }}=\left\|\boldsymbol{P}_{[\mathcal{B}]}\right\|_{1} \underset{\mathcal{H}_{0}}{\stackrel{\mathcal{H}_{1}}{\gtrless}} \xi .
$$

Note that (4) and (5) depend on the noise power and thus may suffer noise power uncertainty. In order to counteract this problem, schemes that compute the ENP can be adopted [13]. In the oversampling scenario the noise power can be estimated from the noise-only bands. Thus, the frequency-domain version of the ENP-ED test is given by

$$
\mathrm{T}_{\text {enp-ed }}=\frac{\left\|\boldsymbol{P}_{[\mathcal{B}]}\right\|_{1}}{\left\|\boldsymbol{P}_{[\overline{\mathcal{B}}]}\right\|_{1}} \underset{\mathcal{H}_{0}}{\stackrel{\mathcal{H}_{1}}{\gtrless}} \xi
$$

where $\boldsymbol{P}_{[\overline{\mathcal{B}}]}$ is the vector containing the periodogram bins that are out of the signal band. Note that the energybased detectors (4)-(6) can be used to infer the presence of a signal in the observed band also with non-flat noise PSD. In this case the tests depend on the noise PSD shape. Considering (6), note that both the numerator and the denominator are proportional to $\sigma^{2}$, and thus $\mathrm{T}_{\text {enp-ed }}$ does not suffer noise uncertainty.

In presence of colored noise, flatness-based detectors can be adopted after a frequency-domain whitening, consisting of defining a vector $\mathbf{Q}$ whose elements are obtained as

$$
Q_{i}=\frac{P_{i}}{W_{i}}
$$

where $W_{i}$ are the elements of $\boldsymbol{W}$ according to (3). In this case, the test (6) becomes

$$
\mathrm{T}_{\text {w-enp-ed }}=\frac{\left\|\mathbf{Q}_{[\mathcal{B}]}\right\|_{1}}{\left\|\mathbf{Q}_{[\overline{\mathcal{B}}]}\right\|_{1}} \underset{\mathcal{H}_{0}}{\stackrel{\mathcal{H}_{1}}{\gtrless}} \xi .
$$

Considering the white noise case, some simple frequency-domain tests proposed measure the "flatness" of the received signal. A flat spectrum is expected, indeed, under $\mathcal{H}_{0}$; otherwise $\mathcal{H}_{1}$ occurs. In presence of colored noise, flatness-based detectors can be adopted after the frequency-domain whitening. For example, the arithmetic-geometric mean ratio test (AGM) [30] becomes

$$
\mathrm{T}_{\text {wf-agm }}=\frac{\frac{1}{N_{\text {fft }}} \sum_{i=1}^{N_{\text {fft }}} Q_{i}}{\prod_{i=1}^{N_{\text {fft }}} Q_{i}^{1 / N_{\text {fft }}}} \underset{\mathcal{H}_{0}}{\stackrel{\mathcal{H}_{1}}{\gtrless}} \xi .
$$

An alternative test that can be adopted in presence of non-flat noise PSD is the following. Under $\mathcal{H}_{0}$ it is 
expected that $\boldsymbol{P}$ exhibits a high degree of similarity with $\boldsymbol{W}$, while they should differ under $\mathcal{H}_{1}$. Therefore, in order to measure the degree of similarity between $\boldsymbol{P}$ and $\boldsymbol{W}$, we propose the test

$$
\mathrm{T}_{\mathrm{fc}}=\frac{\boldsymbol{P}^{\mathrm{T}} \boldsymbol{W}}{\|\boldsymbol{P}\|_{2}\|\boldsymbol{W}\|_{2}} \underset{\mathcal{H}_{1}}{\stackrel{\mathcal{H}_{0}}{\gtrless}} \xi
$$

in which the decision metric is the correlation coefficient between $\boldsymbol{P}$ and $\boldsymbol{W}$. This test resembles the estimator-correlator detector proposed in [33, Section III-B], where the estimated PSD of the received signal is correlated with the known PSD of the signal to be detected. However, note that $\mathrm{T}_{\mathrm{fc}}$ is different, as it infers the presence of any signal present in the observed band without any assumption on the signal to be detected.

\section{TIME-DOMAIN DETECTORS}

Using oversampling, time domain tests generally exploit the correlation properties of $\mathbf{y}$ to distinguish the signal to be detected from white Gaussian noise (WGN). In fact, under $\mathcal{H}_{0}$ the covariance matrix of white noise is $\boldsymbol{\Sigma}_{0}=\mathbb{E}\left\{\mathbf{n} \mathbf{n}^{\mathrm{H}}\right\}=\sigma^{2} \mathbf{I}_{p}$ and thus its eigenvalues are all equal to $\sigma^{2}$. Conversely, under $\mathcal{H}_{1}$, the eigenvalues are not all equal. Based on this, eigenvalue-based tests exploit the eigenvalue spread to discriminate between $\mathcal{H}_{0}$ and $\mathcal{H}_{1}$.

When the covariance matrix of the signal, $\Sigma=$ $\mathbb{E}\left\{\mathbf{y} \mathbf{y}^{\mathrm{H}}\right\}$, is unknown, the most popular detection schemes use the eigenvalues of the sample covariance matrix (SCM) of $\mathbf{y}$ [11], [12], [18], [19]. In this case, the detector collects $N$ snapshots of $\mathbf{y}$ organized in a $p \times n$ matrix ( $n$ and $p$ are such that $N=n p$ )

$$
\mathbf{Y}=\left[\begin{array}{cccc}
y_{1} & y_{p+1} & \ldots & y_{(n-1) p+1} \\
y_{2} & y_{p+2} & \ldots & y_{(n-1) p+2} \\
\ldots & \ldots & \ldots & \ldots \\
y_{p} & y_{2 p} & \ldots & y_{n p}
\end{array}\right]
$$

Then, the eigenvalues of the SCM $\mathbf{S}=\mathbf{Y Y}^{\mathrm{H}} / n$, denoted as $\lambda_{1} \geq \lambda_{2} \geq \cdots \geq \lambda_{p}$, are used as estimate of the eigenvalues of $\boldsymbol{\Sigma}$. Previous works adopt $p=$ OSF and $n=\lfloor N / p\rfloor$ [12], [34]. In this case, the rows of $\mathbf{Y}$ are sequences obtained using a sampling period equal to the symbol time. Assuming that the signal to be detected is composed by independent symbols, if $p=$ OSF the rows of $\mathbf{Y}$ are independent, while columns are correlated. ${ }^{5}$

Two popular eigenvalue-based tests are the sphericity test (also called AGM), which is the generalized likelihood ratio test (GLRT) in additive white Gaussian noise (AWGN) [18], [35], and the locally best invariant (LBI) test [36], which has been shown to outperform

\footnotetext{
${ }^{5}$ In this case the oversampling-based detection problem is equivalent to a multiple antennas or a cooperative spectrum sensing problem.
}

other eigenvalue-based test with colored noise [19]. The corresponding decision metrics are given by

$$
\begin{aligned}
\mathrm{T}_{\mathrm{sph}} & =\frac{\left(\prod_{i=1}^{p} \lambda_{i}\right)^{1 / p}}{\left(\sum_{i=1}^{p} \lambda_{i}\right) / p} \underset{\mathcal{H}_{1}}{\stackrel{\mathcal{H}_{0}}{\gtrless}} \xi \\
\mathrm{T}_{\mathrm{lbi}} & =\frac{\sum_{i=1}^{p} \lambda_{i}^{2}}{\left(\sum_{i=1}^{p} \lambda_{i}\right)^{2}} \underset{\mathcal{H}_{0}}{\stackrel{\mathcal{H}_{1}}{\gtrless}} \xi .
\end{aligned}
$$

In the considered setting, where noise is colored, eigenvalue-based algorithms can be adopted if timedomain whitening is applied to the received samples before detection [11]. ${ }^{6}$ Similarly to the frequency-domain approach, whitening is based on the calibration phase described in Section II, from which the SCM of noise under $\mathcal{H}_{0}, \mathbf{S}_{0}=\mathbf{Y} \mathbf{Y}^{\mathrm{H}} / n$, is obtained. Eigenvalue tests can then be applied considering now the eigenvalues $\lambda_{1}^{\mathbf{B}} \geq \lambda_{2}^{\mathbf{B}} \geq \cdots \geq \lambda_{p}^{\mathbf{B}}$ of $\mathbf{B} \mathbf{S} \mathbf{B}^{\mathrm{H}}$, where $\mathbf{B}$ is the whitening matrix, i.e., a matrix such that $\mathbf{B} \mathbf{S}_{0} \mathbf{B}^{\mathrm{H}}=\mathbf{I}_{p}$. Therefore, the sphericity test in the presence of colored noise becomes

$$
\mathrm{T}_{\mathrm{w}-\mathrm{sph}}=\frac{\left(\prod_{i=1}^{p} \lambda_{i}^{\mathbf{B}}\right)^{1 / p}}{\left(\sum_{i=1}^{p} \lambda_{i}^{\mathbf{B}}\right) / p} \underset{\mathcal{H}_{1}}{\stackrel{\mathcal{H}_{0}}{\gtrless}} \xi
$$

and the LBI test results into

$$
\mathrm{T}_{\mathrm{w}-\mathrm{lbi}}=\frac{\sum_{i=1}^{p}\left(\lambda_{i}^{\mathbf{B}}\right)^{2}}{\left(\sum_{i=1}^{p} \lambda_{i}^{\mathbf{B}}\right)^{2}} \underset{\mathcal{H}_{0}}{\stackrel{\mathcal{H}_{1}}{\gtrless}} \xi .
$$

\section{NUMERICAL RESULTS AND DISCUSSION}

This section presents the receiver operating characteristic (ROC) curves of the detectors described in Sections III and IV, using samples captured from an RTL-SDR dongle. The RTL-SDR receiver is tuned at $430 \mathrm{MHz}$ with sampling frequency $f_{\mathrm{s}}=1 \mathrm{MHz}$. This frequency band has been chosen for being a signalfree band in our laboratory at the Cesena campus of the University of Bologna. The signal to be detected is an orthogonal frequency-division multiplexing (OFDM) waveform transmitting independent symbols and having a $250 \mathrm{kHz}$ bandwidth, generated using a USRP platform. The transmitter has been located in a non-line-of-sight position and its transmit power has been tuned to have a SNR at the receiver equal to $-10 \mathrm{~dB}$.

\footnotetext{
${ }^{6}$ Some papers, such as [12], propose to use eigenvalue-based tests also in presence of colored noise, i.e. when the covariance matrix eigenvalues under $\mathcal{H}_{0}$ are not all equal. This is not a good practice in general. Moreover, the decision regions of the detector can, indeed, depend on the SNR, on the degree of correlation between noise samples, or on other parameters, giving a non-unique criterion for making the decision.
} 


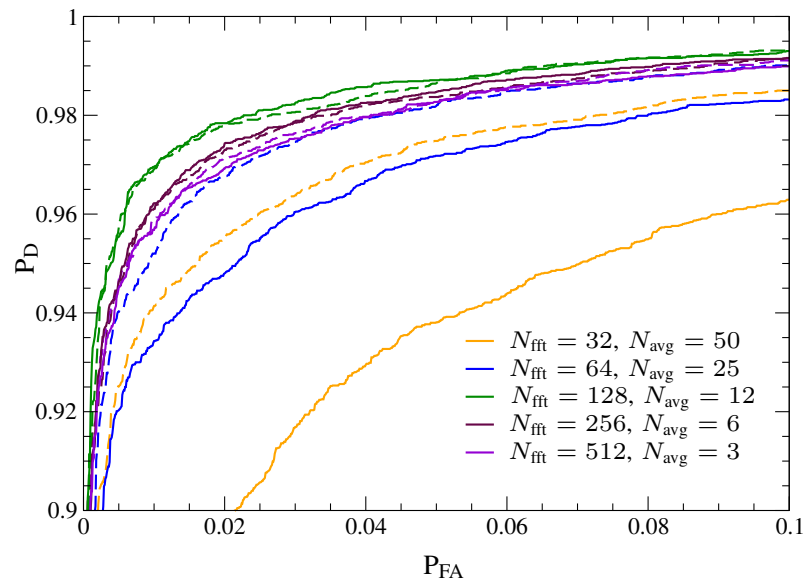

Fig. 2. ROCs for $\mathrm{T}_{\text {enp-ed }}$ (continuous lines) and $\mathrm{T}_{\mathrm{W} \text {-enp-ed (dashed }}$ lines), varying $N_{\text {fft }}$ and $N_{\text {avg }}$ with $N=1600$. Test on real samples captured with RTL-SDR.

\section{A. Parameters setting}

The performance of frequency-domain detectors introduced in Section III depends on the total number of samples collected $N$. Setting $N$, it is possible to trade-off between $N_{\mathrm{fft}}$ and $N_{\text {avg }}$ for the estimation of the PSD in (2). In particular, in the following we set $N=1600$ and we vary $N_{\text {fft }}$ and $N_{\text {avg }}$ pairs such that $N_{\text {avg }}=\left\lfloor N / N_{\text {fft }}\right\rfloor$.

Fig. 2 shows the ROCs for $\mathrm{T}_{\text {enp-ed }}$ (continuous lines) and $\mathrm{T}_{\text {w-enp-ed }}$ (dotted lines), for different $N_{\mathrm{fft}}, N_{\mathrm{avg}}$ pairs. The parameter $N_{\mathrm{fft}}$ impacts the frequency resolution of the PSD estimate. A small $N_{\mathrm{fft}}$ implies, indeed, to have just few DFT elements each of which collects a large contribution from sidelobes. Therefore, increasing $N_{\text {fft }}$ provides a better estimate of the in-band signal energy. Considering $N_{\text {avg }}$, note that it corresponds to the number periodograms averaged in (2) and thus it impacts the accuracy of the PSD estimate. Therefore, there is a trade-off between these two parameters that provide the best performance. From Fig. 2 we can see that the best choice is $N_{\mathrm{fft}}=128$ and $N_{\mathrm{avg}}=12$. Note that increasing $N_{\mathrm{fft}}$ above this level, the detector performance decreases due to the small number of averages $N_{\text {avg }}$. Regarding the comparison between the whitened and non-whitened ED, the plots confirm the advantage introduced by frequency-domain whitening, except for the cases where near-optimal values of $N_{\mathrm{fft}}$ and $N_{\text {avg }}$ are chosen. In these cases whitening does not provide appreciable improvements.

In Fig. 3 the ROCs for $\mathrm{T}_{\mathrm{fc}}$ (continuous line) and $\mathrm{T}_{\mathrm{wf}-\mathrm{agm}}$ (dashed lines) are reported for different combinations of $N_{\mathrm{fft}}$ and $N_{\mathrm{avg}}$, respectively. From the comparison, we can see that decreasing $N_{\text {fft }}$ provides a higher probability of detection.

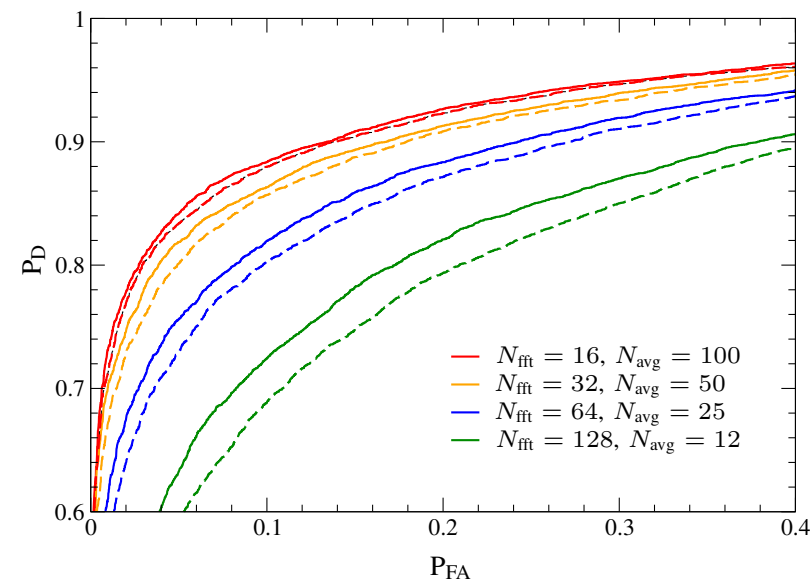

Fig. 3. ROCs for the frequency-based detectors $T_{f c}$ (continuous) and $\mathrm{T}_{\text {wf-agm }}$ (dashed), with $N=1600$. Test on real samples captured with RTL-SDR.

\section{B. Detection performance comparison}

Fig. 4 shows the comparison between the ROCs of the frequency-based detectors introduced in Section III and the time-domain tests described in Section IV. We adopt in this case the parameters $N_{\mathrm{fft}}$ and $N_{\mathrm{avg}}$ that maximize the detection performance for each test. From the comparison, we can clearly see that frequency-domain detectors outperform eigenvalue-based tests. Note, in particular, that $\mathrm{T}_{\text {enp-ed }}$ provides the best performance with much higher detection probability with respect to other detectors. For example, when $\mathrm{P}_{\mathrm{FA}}=0.01$ the probability of detection of $\mathrm{T}_{\text {enp-ed }}$ is approximately $\mathrm{P}_{\mathrm{D}}=0.98$, while for $\mathrm{T}_{\mathrm{fc}}$ is below $\mathrm{P}_{\mathrm{D}}=0.8$ and for $\mathrm{T}_{\mathrm{w}-\mathrm{sph}}$ is below $\mathrm{P}_{\mathrm{D}}=0.7$.

The large detection performance gain of the ENP-ED can be explained considering the fact that $\mathrm{T}_{\text {enp-ed }}$ exploits an additional information with respect to other detectors. Note, indeed, that $\mathrm{T}_{\text {enp-ed }}$ requires the knowledge of the signal band, $\mathcal{B}$. This is a valid assumption for example when the signal to be detected is a primary user (PU), whose channelization is generally known from standards and regulations.

In different $\mathrm{CR}$ scenarios, however, $\mathcal{B}$ is unknown, and the best choice is to adopt $\mathrm{T}_{\mathrm{fc}}$, which does not require any knowledge of the signal to be detected (including its operating band) and that provides better detection performance than $\mathrm{T}_{\mathrm{wf}-\mathrm{agm}}$ and eigenvalues-based tests.

\section{CONCLUSION}

In this paper we discussed the problem of SS in the presence of front-end impairments, typical of inexpensive devices. We verified that the receiver noise is well described by a non-white Gaussian process with time-varying power. Based on this model we proposed two frequency-domain detectors, and showed that they outperform commonly adopted time-domain eigenvaluebased tests. 


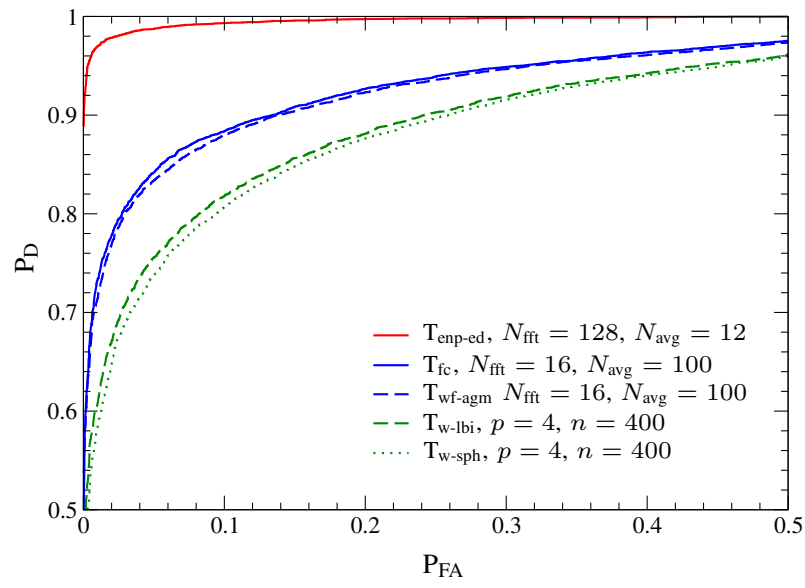

Fig. 4. ROC comparison with $N=1600$. Samples captured with RTL-SDR.

\section{REFERENCES}

[1] National Spectrum Consortium. [Online]. Available: https://www.nationalspectrumconsortium.org

[2] G. Sklivanitis, A. Gannon, S. N. Batalama, and D. A. Pados, "Addressing next-generation wireless challenges with commercial software-defined radio platforms," IEEE Commun. Mag., pp. 59-67, Jan. 2016.

[3] J. Mitola, "Software radios: Survey, critical evaluation and future directions," IEEE Aerosp. Electron. Syst. Mag., vol. 8, no. 4, pp. 25-36, Apr. 1993

[4] A. A. Abidi, "The path to the software-defined radio receiver," IEEE J. Solid-State Circuits, vol. 42, no. 5, pp. 954-966, May 2007.

[5] K. Sithamparanathan and A. Giorgetti, Cognitive Radio Techniques: Spectrum Sensing, Interference Mitigation and Localization. Boston, USA: Artech House Publishers, Nov. 2012.

[6] Ettus Research. Universal Software Radio Peripheral. [Online]. Available: https://www.ettus.com/product

[7] R. W. Stewart, K. W. Barlee, D. S. W. Atkinson, and L. H. Crockett, Software Defined Radio using MATLAB \& Simulink and the RTL-SDR, 1st ed. Glasgow, Scotlabd, UK: Wiley, 2015.

[8] A. Mariani, S. Kandeepan, and A. Giorgetti, "Periodic spectrum sensing with non-continuous primary user transmissions," IEEE Trans. Wireless Commun., vol. 14, no. 3, pp. 1636-1649, Mar. 2015.

[9] A. Mariani, A. Giorgetti, and M. Chiani, "Wideband spectrum sensing by model order selection," IEEE Trans. Wireless Commun., vol. 14, no. 12, pp. 6710-6721, Dec. 2015.

[10] R. N. McDonough and A. Whalen, Detection of signals in noise. Academic Press, 1995.

[11] Y. Zeng and Y.-C. Liang, "Eigenvalue-based spectrum sensing algorithms for cognitive radio," IEEE Trans. Commun., vol. 57, no. 6, pp. 1784-1793, Jun. 2009.

[12] S. K. Sharma, S. Chatzinotas, and B. Ottersten, "Eigenvaluebased sensing and SNR estimation for cognitive radio in presence of noise correlation," IEEE Trans. Veh. Technol., vol. 62, no. 8, pp. 3671-3684, Oct. 2013.

[13] A. Mariani, A. Giorgetti, and M. Chiani, "Effects of noise power estimation on energy detection for cognitive radio applications," IEEE Trans. Commun., vol. 59, no. 12, pp. 3410-3420, Dec. 2011.

[14] D. Torrieri, "The radiometer and its practical implementation," in Proc. IEEE Military Commun. Conf. (MILCOM 2010), Oct./Nov. 2010, pp. 304-310.

[15] A. Sonnenschein and P. M. Fishman, "Radiometric detection of spread-spectrum signals in noise of uncertain power," IEEE Trans. Aerosp. Electron. Syst., vol. 28, no. 3, pp. 654-660, Jul. 1992.
[16] A. Mariani, A. Giorgetti, and M. Chiani, "Wideband spectrum sensing by model order selection," IEEE Trans. Wireless Commun., vol. 14, no. 12, pp. 6710-6721, Dec. 2015.

[17] A. Mariani, S. Kandeepan, and A. Giorgetti, "Periodic spectrum sensing with non-continuous primary user transmissions," IEEE Trans. Wireless Commun., vol. 14, no. 3, pp. 1636-1649, Mar. 2015.

[18] A. Mariani, A. Giorgetti, and M. Chiani, "Test of independence for cooperative spectrum sensing with uncalibrated receivers," in Proc. IEEE Global Commun. Conf. (GLOBECOM 2012), Anaheim, CA, USA, Dec. 2012.

[19] L. Wei, O. Tirkkonen, and Y.-C. Liang, "Multi-source signal detection with arbitrary noise covariance," IEEE Trans. Signal Process., vol. 62, no. 22, pp. 5907-5918, Nov. 2014.

[20] A. Mariani, A. Giorgetti, and M. Chiani, "Recent advances on wideband spectrum sensing for cognitive radio," in Cognitive Communications and Cooperative HetNet Coexistence, Signals and Communication Technology (M.-G. Di Benedetto and F. Bader, Eds.). Switzerland: Springer Int. Pub., 2014, ch. 1.

[21] E. H. Gismalla and E. Alsusa, "On the performance of energy detection using bartlett's estimate for spectrum sensing in cognitive radio systems," IEEE Trans. on Signal Proc., vol. 60, no. 7, pp. 3394-3404, July 2012.

[22] J. Verlant-Chenet, J. Renard, J. M. Dricot, P. D. Doncker, and F. Horlin, "Sensitivity of spectrum sensing techniques to RF impairments," in 2010 IEEE 71 st Vehicular Technology Conference, May 2010 , pp. $1-5$

[23] A. Zahedi-Ghasabeh, A. Tarighat, and B. Daneshrad, "Cyclostationary sensing of OFDM waveforms in the presence of receiver RF impairments," in IEEE Wireless Comm. and Netw. Conf., April 2010, pp. 1-6.

[24] W. Han, C. Huang, J. Li, Z. Li, and S. Cui, "Correlation based spectrum sensing with over-sampling in cognitive radio," IEEE J. Sel. Areas Commun., vol. 33, no. 5, May 2015.

[25] B. Yazici and S. Yolacan, "A comparison of various tests of normality," Journal of Statistical Computation and Simulation, vol. 77, no. 2, pp. 175-183, Feb. 2007.

[26] M. A. Stephens, "EDF statistics for goodness of fit and some comparisons," Journal of the American Statistical Association, vol. 69, no. 347, pp. 730-737, Sep. 1974.

[27] N. M. Razali and Y. B. Wah, "Power comparisons of ShapiroWilk, Kolmogorov-Smirnov, Lilliefors and Anderson-Darling tests," Journal of Statistical Modeling and Analytics, vol. 2, no. 1, pp. 21-33, 2011.

[28] S. A. Andersson and M. D. Perlman, "Two testing problems relating the real and complex multivariate normal distributions," Journal of Multivariate Analysis, vol. 15, no. 1, pp. 21-51, 1984.

[29] T. Adali, P. J. Schreier, and L. L. Scharf, "Complex-valued signal processing: The proper way to deal with impropriety," Signal Processing, IEEE Transactions on, vol. 59, no. 11, pp. 51015125, Nov. 2011

[30] H. Cao and J. Peissig, "Practical spectrum sensing with frequency-domain processing in cognitive radio," in Proc. European Signal Process. Conf. (EUSIPCO 2012), Bucharest, Romania, Aug. 2012, pp. 435-439.

[31] D. Borio, E. Angiuli, R. Giuliani, and G. Baldini, "Robust spectrum sensing demonstration using a low-cost front-end receiver," Int. J. Antenn. Propag., 2015.

[32] J. G. Proakis and D. G. Manolakis, Digital Signal Processing: Principles, Algotithms, and Applications, 3rd ed. Prentice Hall, 1996.

[33] H. V. Poor, An introduction to signal detection and estimation. Springer Science \& Business Media, 1994.

[34] F. Penna, R. Garello et al., "Cooperative spectrum sensing based on the limiting eigenvalue ratio distribution in wishart matrices," IEEE Commun. Lett., vol. 13, no. 7, pp. 507-509, Jul. 2009.

[35] J. Mauchly, "Significance test for sphericity of a normal n-variate distribution," The Annals of Mathematical Statistics, vol. 11, no. 2, pp. 204-209, Jun. 1940.

[36] S. John, "Some optimal multivariate tests," Biometrika, vol. 58, no. 1, pp. 123-127, Apr. 1971. 